\title{
BMJ Open Epidemiology of major depressive disorder in South Africa (1997-2015): a systematic review protocol
}

\author{
Mweete D Nglazi, ${ }^{1}$ Jané D Joubert, ${ }^{1}$ Dan J Stein, ${ }^{2,3}$ Crick Lund, ${ }^{4,5}$ \\ Charles S Wiysonge, ${ }^{6,7}$ Theo Vos, ${ }^{8}$ Victoria Pillay-van Wyk, ${ }^{1}$ Rifqah A Roomaney, ${ }^{1}$ \\ Lorrein S Muhwava, ${ }^{9}$ Debbie Bradshaw ${ }^{1}$
}

To cite: Nglazi MD, Joubert JD, Stein DJ, et al. Epidemiology of major depressive disorder in South Africa (1997-2015): a systematic review protocol. BMJ Open 2016;6: 011749 . doi:10.1136/bmjopen-2016011749

- Prepublication history for this paper is available online To view these files please visit the journal online (http://dx.doi.org/10.1136/ bmjopen-2016-011749)

Received 1 March 2016 Revised 10 May 2016 Accepted 26 May 2016
CrossMark

For numbered affiliations see end of article.

Correspondence to Mweete D Nglazi; mweete.nglazi@mrc.ac.za

\section{ABSTRACT}

Introduction: Major depressive disorder (MDD) is a leading cause of disease and disability globally and in South Africa. Epidemiological data for MDD are essential to estimate the overall disease burden in a country. The objective of the systematic review is to examine the evidence base for prevalence, incidence, remission, duration, severity, case fatality and excess mortality of MDD in South Africa from 1997 to 2015.

Methods and analysis: We will perform electronic searches in PubMed, PsycINFO, Scopus and other bibliographical databases. Articles published between January 1997 and December 2015 will be eligible for inclusion in this review. The primary outcomes will be prevalence, incidence, remission, duration, severity, case fatality and excess mortality of MDD. The secondary outcomes will be risk factors and selected populations for MDD. If appropriate, a meta-analysis will be performed. If a meta-analysis is not possible, the review findings will be presented narratively and in tables. Subgroup analyses will be conducted with subgroups defined by population group, rural/urban settings and study designs, if sufficient data are available.

Ethics and dissemination: The systematic review will use published data that are not linked to individuals. The review findings may have implications for future research prioritisation and disease modelling of MDD to estimate its morbidity burden in South Africa, and will be disseminated electronically and in print through peerreviewed publications.

Trial Registration number: International Prospective Register of Systematic Reviews (PROSPERO) CRD42015024885.

\section{INTRODUCTION}

Major depressive disorder (MDD), a common mental disorder, is among the leading three causes of disease burden globally and in South Africa. Globally, the prevalence of MDD is estimated to have increased significantly by $53 \%$ from 1990 to reach over 253 million prevalent cases in 2013. On a similar scale, the years lived with disability (YLDs) for MDD globally increased

\section{Strengths and limitations of this study}

- To the best of our knowledge, this is the first systematic review to assess the evidence base on the epidemiological parameters and risk factors for major depressive disorder (MDD) in South Africa.

- The study is likely to provide epidemiological parameters commonly used in modelling health conditions with the purpose of estimating the morbidity burden in a country, and to subsequently help guide resource prioritisation, decision-making and policy development.

- This protocol complies with the 'Preferred Reporting Items for Systematic Review and Meta-Analysis Protocols (PRISMA-P) 2015' guidelines.

- There are several assessment tools to measure MDD which may limit comparison across studies.

- Observational studies with a high risk of bias may present a limitation to the study. We will, however, investigate the robustness of the methods and results by using a risk of bias tool and excluding studies with a high risk of bias.

significantly by $53 \%$ from 1990 to reach 52 million YLDs in 2013. ${ }^{1}$ In South Africa, too, the YLDs for MDD increased significantly by $58 \%$ from 1990 to reach 408578 YLDs in $2013 .^{2}$ Much of the increases arose from the growth and ageing of the population as well as the widespread under-resourcing of mental health services in South Africa, resulting in underdiagnoses and undertreatment of mental disorders (including MDD). ${ }^{3}$

The first port of entry for South Africans is often the primary healthcare setting. ${ }^{4}$ However, the literature shows that there are barriers to help-seeking and treatmentseeking for mental illnesses. ${ }^{5-7}$ Reasons in the literature include stigma, low health literacy levels (in terms of knowledge of the disease and its treatment) and financial 
difficulties. ${ }^{6}$ In addition, comorbidity complicates helpseeking and treatment-seeking for mental illness. ${ }^{5} 7$ Moreover, mental disorders (including MDD) often go unrecognised by healthcare providers and therefore untreated in primary health settings. ${ }^{8}$ They are disabling and associated with a significant economic burden at both the individual and societal levels, resulting from a reduction in health-related quality of life, disrupted work and life roles, as well as increased morbidity and mortality. ${ }^{4-11}$

Health in South Africa is characterised by an enormous and diverse burden of disease $\mathrm{e}^{12-15}$ and comorbidity of MDD with a range of other conditions is a reason for concern. MDD, for example, is comorbid with infectious diseases, such as HIV/AIDS, ${ }^{7}$ which are pandemic in the country. MDD is also associated with noncommunicable diseases, ${ }^{16}$ high rates of violence and injury $^{17}$ and maternal and child illness. ${ }^{18}$ In addition, South Africans may be at risk for neuropsychiatric disorders given the high levels of unemployment and poverty, ${ }^{19}$ high rates of crime, inadequate social services and other potentially stressful living conditions such as poor and unstable housing. ${ }^{20}$ Moreover, evidence suggests that MDD is comorbid with a variety of psychiatric conditions and medical conditions as well as substance use disorders. ${ }^{4}{ }^{21}$ It is therefore reasonable to argue that, with the high burden of disease,,$^{13} 22{ }^{23}$ and shortages in healthcare resources and the potentially adverse living conditions in South Africa, ${ }^{12}{ }^{24-26}$ MDD is likely to be highly prevalent.

MDD in the Diagnostic and Statistical Manual of Mental Disorders, Fifth Edition (DSM-V) ${ }^{27}$ is characterised by one or more major depressive episodes, lasting for at least 2 weeks. This closely resembles the criteria for recurrent depressive disorder in the existing International Statistical Classification of Diseases and Related Health Problems, 10th Revision (ICD-10). ${ }^{28}$ An MDD episode involves symptoms of depressed mood and/or loss of interest or pleasure in all or most activities, occurring most of the day and nearly every day, plus at least five of the following symptoms: appetite or weight change, sleep disturbances, restlessness, fatigue, feelings of guilt or worthlessness, impaired concentration and suicidal ideation. ${ }^{29}$ The DSM-V criteria for MDD have remained mostly unchanged from the fourth edition of the text revision of the DSM, (DSM-IV-TR). DSM-V has eliminated the bereavement exclusion and acknowledged the mixed features of MDD. ${ }^{30}$ In addition, the proposed 11th revision of the ICD criteria for recurrent depressive disorder may differ from those in ICD-10. The suggestion has been made to use only one overall depressive episode diagnosis, with the distinction between first and recurrent episode to become a subtype or be abandoned. ${ }^{31}$

Diagnosis of MDD as per the criteria outlined in the DSM-V and DSM-IV-TR involves establishing the severity of symptoms and their duration as well as the degree of impairment. ${ }^{4} 32$ There are a variety of assessment instruments that may be useful in providing an indication of the severity of symptoms and assessing the severity of symptoms over a specified time period. ${ }^{42}$ These range from self-administered assessment instruments to interviewer-administered assessment instruments. ${ }^{32}$ Furthermore, diagnosis is guided by both a patient history and examination to rule out any underlying comorbid psychiatric disorder, substance use disorder and general medical conditions. ${ }^{42}$ Finally, the literature indicates that diagnostic criteria are useful to follow for the diagnosis of MDD but must be applied with clinical judgement, because the symptoms of depression may vary across gender, age and cultural group. ${ }^{32}$

The second South African National Burden of Disease Study, which estimates the disease burden for the country using different summary measures of population health, including the disability-adjusted life year (DALY), is currently underway. The DALY combines the health loss from premature mortality (or years of life lost (YLLs)) and the loss of healthy life from disability associated with non-fatal outcomes (or YLDs). An essential prerequisite for estimating the DALY is the availability of nationally representative epidemiological data. We therefore aim to assess the evidence base for prevalence, incidence, remission, duration, severity, case fatality and excess mortality of MDD in South Africa from 1997 to 2015. A secondary aim is to identify risk factors for MDD.

\section{METHODS}

\section{Criteria for included studies}

\section{Types of studies}

We will include population-based surveys, prospective or retrospective cohort, case-control and cross-sectional studies published in English, with more than 100 participants, that reported on the epidemiological parameters of interest in the general population and in specific populations such as those with chronic diseases (including HIV), pregnancy and those exposed to trauma or negative life experiences. Studies that report on cases which met diagnostic criteria for MDD as described in the DSM-V/DSM-IV-TR or ICD-10 will be included. Studies in which a measurement tool was used to screen for and diagnose MDD will also be included.

For prevalence, we will include studies reporting point (ie, current or past month prevalence) and period prevalence (ie, 6-month and/or 12-month prevalence). For incidence, we will include studies reporting on cumulative incidence rates (ie, number of new cases in a specified time period in a population at risk) or incidence rates (ie, number of new cases over total personyears of follow-up susceptible) ${ }^{33}$ For remission, we will include studies reporting on a rescinded diagnosis of MDD (ie, DSM-V/DSM-IV-TR or ICD-10 criteria no longer met). For duration, we will include studies reporting on time to end of a major depressive episode. ${ }^{34}$ For severity, we will include studies reporting on severity (ie, mild, moderate or severe) based on DSM-V/DSM-IV-TR 
or ICD-10 specifiers. For case fatality, we will include studies reporting on the percentage of persons diagnosed as having MDD who die as a result of the disease within a given time period; ${ }^{35}$ and for excess mortality, we will include studies reporting on relative risk (ie, deaths in individuals with MDD compared with individuals without MDD) or standardised mortality ratios (ie, deaths in individuals with MDD compared with deaths in the total population). ${ }^{33}$

In addition, studies examining the reliability and/or validity of depression measurement tools will be selected for inclusion if they meet all the aforementioned inclusion criteria. Studies using symptom-based instruments that mapped on the criteria proposed by DSM-V/ DSM-IV-TR and ICD-10 will also be included. Randomised controlled trials, published in English, will be included in cases where both the experimental and control groups are selected from the same source population or the larger population of which they are ideally a representative sample.

Studies reporting on depressive symptoms, not specific to MDD, will be excluded. For prevalence, lifetime estimates will be excluded, as recall bias invalidates them as credible measures of disease burden. ${ }^{33}$ Finally, studies that report secondary outcomes and no primary outcomes will be excluded. The primary and secondary outcomes are listed in the 'types of outcome measure' section below.

\section{Types of participants}

We will include studies conducted on persons living in South Africa, regardless of age, gender and study setting. Studies conducted exclusively on recent immigrants or refugees will be excluded because they have a very different risk profile for major depression (usually elevated) compared with the non-immigrants ${ }^{36-39}$ and therefore will not be representative of the broader South African population. ${ }^{40}$

\section{Types of outcome measures}

Primary outcomes

Prevalence: Point prevalence (ie, current or past month prevalence), 6-month or 12-month period prevalence of MDD.

Incidence: Cumulative incidence or incidence rate of a major depressive episode (ie, number of new cases over total person-years of follow-up susceptible).

Remission: Remission rate (ie, percentage of 'untreated' participants remitted at one or more follow-up time-points in the study).

Duration: Average or median duration of a major depressive episode.

Severity: Severity criteria (ie, mild, moderate or severe) of a major depressive episode according to DSM-V/ DSM-IV-TR or ICD-10.

Case fatality: Case fatality rate (ie, percentage of persons diagnosed as having MDD who die as a result of the disease within a specified time period).
Excess mortality: Relative risk (ie, deaths in individuals with MDD compared with individuals without MDD) or standardised mortality ratios (ie, deaths in individuals with MDD compared with deaths in the total population).

\section{Secondary outcome}

Risk factors for MDD. Risk factors for this mood disorder cited in the literature are sociodemographic factors (such as age, gender, occupation, educational level, marital status, employment status, ethnicity, socioeconomic status), familial factors (such as family history of MDD, family conflict), stressful life events (eg, death, assault, marital discord, divorce), comorbidity (eg, substance use disorder, psychiatric disorders and medical illnesses) and early trauma (eg, early childhood maltreatment; early prolonged emotional, physical and sexual abuse and early death of a parent). ${ }^{41} 42$

\section{Search strategy}

The search strategy aims to find published articles written in English, that described the prevalence, incidence, remission, duration, severity of, case fatality or excess mortality of MDD, that were conducted from January 1997 up to December 2015. The electronic databases that will be searched are CINAHL, JTSOR, Popline, PubMed, Science Direct, Scopus, Web of Science, PsycINFO, PsychARTICLES and PsychBooks (table 1). The time period was chosen because we have YLL estimates for this period which will be combined with the YLD estimates obtained through disease modelling to calculate the DALY.

Experts in the field will be consulted to inform about possible non-journal literature and local data sources. We will also check reference lists of included studies and other relevant publications for additional studies.

\section{Study selection}

The titles and abstracts of the search output will be screened independently by two review authors in order to identify potentially eligible studies. Full-text articles of

Table 1 PubMed search strategy, modified as appropriate for use in other databases

\section{Search Query}

\#4 Search ((\#3 NOT (animals[mh] NOT humans [mh]))) AND ("1997/01/01"[Date-Publication] : "2015/12/31"[Date-Publication])

\#3 Search (\#1 AND \#2)

\#2 Search (South Africa[mh] OR South Africa*[tiab] OR RSA[tiab] OR Africa, Southern[mh:noexp] OR Southern Africa[tiab])

\#1 Search (Depression[Mesh] OR "Depressive Disorder"[Mesh] OR "Depressive Disorder, Major"[Mesh] OR "major depression"[tiab] OR "major depressive disorder"[tiab] OR dysthymi* [tiab]) 
these studies will be obtained and two authors will independently examine these for inclusion in the review using prespecified criteria. Any disagreements will be resolved by discussion and consensus involving the two authors, or a third review author who will arbitrate, if needed.

\section{Data extraction}

A standardised data extraction form will be used for data extraction. Two authors will independently perform data extraction. Any disagreements arising during the data extraction process will be resolved by discussion and consensus involving the two authors or a third review author will arbitrate, if needed. Information will be extracted regarding study title, author(s), year of study and publication; study design/data source; population characteristics such as age and sex; study setting and geographic location (such as rural, urban); details of outcome measures; and details necessary to assess the risk of bias. Authors of reports/articles will be contacted for additional information on unclear or missing data.

\section{Risk of bias assessment}

Two authors will independently assess the risk of bias and methodological quality for each study using a piloted checklist ${ }^{43}$ adapted from the risk of bias tool for population-based studies described by Hoy et $a l^{44}$ and the Newcastle-Ottawa Scale for assessing the quality of nonrandomised studies. ${ }^{45}$ The dimensions covered in the checklist include external validity (ie, representativeness, non-response bias and loss to follow-up) and internal validity (ie, case definition, measurement of cases and consistency of measurement of cases, uncertainty of estimation, appropriateness of time factor for outcome measure, appropriateness of numerator and denominator in calculation of estimate and confounding) ${ }^{43}$ This will be assessed qualitatively in terms of the anticipated impact on bias. In addition, total scores of the risk of bias will be taken into account which range from 1 to 20: a score of 1-6 will be rated high risk, 7-13 moderate risk, and 14-20 low risk. ${ }^{43}$ Differences between the two authors will be resolved by discussion and consensus and a third author will arbitrate, if needed.

\section{Data synthesis}

Separate meta-analyses will be performed for quantitative data on prevalence, incidence, remission, duration, severity, case fatality and excess mortality for MDD from studies rated as low or moderate risk using STATA 13 (StataCorp, College Station, Texas, USA). We will combine the study-specific estimates and obtain the overall summary estimate and 95\% CI across studies. Clinical heterogeneity will be investigated by looking at the types of participants in each study. The $\chi^{2}$ test will be used to identify statistically significant heterogeneity. The latter will be considered to exist when $\chi^{2}$ $\mathrm{p} \leq 0.10{ }^{46}{ }^{47}$ The $\mathrm{I}^{2}$ statistic will be used to evaluate the degree of heterogeneity. If the study results are found to be statistically homogeneous (ie, when $\chi^{2} \mathrm{p}>0.10$ ), we will pool them using the fixed-effect meta-analysis. Otherwise, we will use random effects meta-analysis. Potential publication bias will be assessed by visual inspections of funnel plots. ${ }^{48}$ Where studies are found to be clinically and statistically heterogeneous, we will conduct a narrative synthesis including tables and figures. We anticipate that the paucity of published studies reporting on incidence, remission, duration, severity, case fatality and excess mortality of MDD and the heterogeneous nature of the reported results will preclude meta-analysis. In this event, only a narrative synthesis of results will be conducted.

For risk factors for MDD, in anticipation of the large variability in their investigation and reporting across studies, only a narrative synthesis of the evidence will be conducted, including tables. For studies rated low or moderate risk, we will report the measure of association used for each of the risk factors investigated with their corresponding $95 \% \mathrm{CI}$ and indicate whether those measures were adjusted for confounders or not. Further, we will document the number of risk factors reported to be associated with MDD and tabulate the most commonly reported risk factors for MDD.

\section{Analysis of subgroups or subsets}

Where sufficient data are available, subgroup analyses will be performed by population group where identified, MDD assessment instrument, rural/urban settings, and study designs.

\section{Confidence in cumulative evidence}

The grading of recommendations, assessment, development and evaluation (GRADE) approach will be used to assess the certainty of the body of evidence. ${ }^{49} 50$ This method results in the assessment of the certainty of the body of evidence as high, moderate, low or very low. The evidence is considered of high certainty if further research very unlikely to change our confidence in the effect estimate; and moderate certainty if further research is likely to have an important impact on our confidence in the estimate of the effect and may change the estimate. The low certainty evidence implies that further research is likely to have an important impact on our confidence in the effect estimate and may change the estimate, and very low certainty implies that we have very little confidence in the effect estimate and there is uncertainty in the effect estimates.

\section{ETHICS AND DISSEMINATION}

The systematic review does not require ethics approval because published studies with non-identifiable data will be used. None of the data can be linked to an individual. This protocol complies with the 'Preferred Reporting Items for Systematic Review and Meta-Analysis Protocols (PRISMA-P)' guidelines ${ }^{51}$ and any amendments that prove necessary will be tracked, documented and reported transparently. In addition, the findings of 
the systematic review will be reported according to the PRISMA statement, ${ }^{52}$ and will have important implications for epidemiological modelling and research. The review will shed light on the evidence base for epidemiological studies on MDD and the availability of nationally representative local epidemiological data which are needed for the disease modelling of MDD to estimate YLDs; ultimately, these estimates will be combined with YLLs to calculate the DALYs which will inform policymakers and funding bodies regarding decisions on where to focus policy, service and research planning and implementation. Also, the findings may influence future studies to improve study methodology. For example, the use of similar assessment instruments to diagnose MDD will allow for effective comparisons across studies.

\section{Author affiliations}

${ }^{1}$ Burden of Disease Research Unit, South African Medical Research Council, Cape Town, South Africa

${ }^{2}$ Department of Psychiatry \& Mental Health, University of Cape Town, Cape Town, South Africa

${ }^{3} \mathrm{MRC}$ Unit on Anxiety \& Stress Disorders, Department of Psychiatry, Stellenbosch University, Cape Town, South Africa

${ }^{4}$ Alan Alan J Flisher Centre for Public Mental Health, Department of Psychiatry and Mental Health, University of Cape Town, Cape Town, South Africa

${ }^{5}$ Centre for Global Mental Health, Institute of Psychiatry, Psychology and Neuroscience, King's College London, London, UK

${ }^{6}$ Centre for Evidence-based Health Care, Faculty of Medicine and Health Sciences, Stellenbosch University, Cape Town, South Africa

${ }^{7}$ Cochrane South Africa, South African Medical Research Council, Cape Town, South Africa

${ }^{8}$ University of Washington, Institute for Health Metrics and Evaluation, Seattle, Washington, USA

${ }^{9}$ Chronic Diseases Initiative for Africa, University of Cape Town, Cape Town, South Africa

Twitter Follow Charles Wiysonge at @CharlesShey

Contributors MDN conceived the idea, and MDN, JDJ and DB conceptualised the study protocol. MDN, JDJ and DB planned and designed the study protocol. DJS, CL, TV and CSW gave critical insights. MDN wrote the first draft of the study protocol. JDJ and DB critically reviewed the first draft for intellectual input, and all the authors gave inputs into subsequent drafts. VP-vW and MDN participated in the development of the search strategy. VP-vW, RAR, MDN, JDJ and DB planned the data extraction. All the authors have given their approval for publication.

Funding This work was supported by the South African Medical Research Council in terms of the MRC's Flagship Awards Project grant number SAMRC-RFA-IFSP-01-2013/ SA CRA 2.

Disclaimer The funders had no role in the study design, decision to publish or preparation of the manuscript.

Competing interests None declared.

Provenance and peer review Not commissioned; externally peer reviewed.

Open Access This is an Open Access article distributed in accordance with the Creative Commons Attribution Non Commercial (CC BY-NC 4.0) license, which permits others to distribute, remix, adapt, build upon this work noncommercially, and license their derivative works on different terms, provided the original work is properly cited and the use is non-commercial. See: http:// creativecommons.org/licenses/by-nc/4.0/

\section{REFERENCES}

1. Global Burden of Disease Study 2013 Collaborators. Global, regional, and national incidence, prevalence, and years lived with disability for 301 acute and chronic diseases and injuries in 188 countries, 1990-2013: a systematic analysis for the Global Burden of Disease Study 2013. Lancet 2015;386:743-800.

2. Institute for Health Metrics and Evaluation (IHME). GBD compare. Secondary GBD compare. 2015. http://vizhub.healthdata.org/ gbd-compare (accessed 5 May 2015).

3. Lund C, Stein DJ, Flisher AJ, et al. Challenges faced by South African health services in implementing the Mental Health Care Act. S Afr Med J 2007;97:352-3.

4. Freeman CP, Joska JA. Dealing with major depression. $S$ Afr Pharm J 2013;80:16-21.

5. Lund C, Stein DJ, Corrigall J, et al. Mental health is integral to public health: a call to scale up evidence-based services and develop mental health research. S Afr Med J 2008;98:444, 46.

6. Andersson LM, Schierenbeck I, Strumpher J, et al. Help-seeking behaviour, barriers to care and experiences of care among persons with depression in Eastern Cape, South Africa. J Affect Disord 2013;151:439-48.

7. Prince M, Patel V, Saxena S, et al. No health without mental health. Lancet 2007;370:859-77.

8. Petersen I, Lund C. Mental health service delivery in South Africa from 2000 to 2010: one step forward, one step back. S Afr Med J 2011;101:751-7.

9. Lohoff FW. Overview of the genetics of major depressive disorder. Curr Psychiatry Rep 2010;12:539-46.

10. Lund C, Myer L, Stein DJ, et al. Mental illness and lost income among adult South Africans. Soc Psychiatry Psychiatr Epidemiol 2013;48:845-51.

11. Mall S, Lund C, Vilagut G, et al. Days out of role due to mental and physical illness in the South African stress and health study. Soc Psychiatry Psychiatr Epidemiol 2015;50:461-8.

12. Coovadia $H$, Jewkes $R$, Barron $P$, et al. The health and health system of South Africa: historical roots of current public health challenges. Lancet 2009;374:817-34.

13. Bradshaw D, Groenewald P, Laubscher R, et al. Initial burden of disease estimates for South Africa, 2000. S Afr Med J 2003;93:682-8.

14. Bradshaw D, Nannan N, Groenewald P, et al. Provincial mortality in South Africa, 2000-priority-setting for now and a benchmark for the future. S Afr Med J 2005;95:496-503.

15. Chopra M, Lawn JE, Sanders D, et al. Achieving the health Millennium Development Goals for South Africa: challenges and priorities. Lancet 2009;374:1023-31.

16. Mayosi BM, Flisher AJ, Lalloo UG, et al. The burden of noncommunicable diseases in South Africa. Lancet 2009;374:934-47.

17. Seedat $M$, Van Niekerk $A$, Jewkes $R$, et al. Violence and injuries in South Africa: prioritising an agenda for prevention. Lancet 2009;374:1011-22.

18. Chopra M, Daviaud E, Pattinson R, et al. Saving the lives of South Africa's mothers, babies, and children: can the health system deliver? Lancet 2009;374:835-46.

19. Lund C, Kleintjes S, Kakuma R, et al. Public sector mental health systems in South Africa: inter-provincial comparisons and policy implications. Soc Psychiatry Psychiatr Epidemiol 2010;45:393-404.

20. Moosa MYH, Jeenah FY. Treating depression in HIV/AIDS. S Afr $J$ Psychiatry 2007;13:86-8.

21. Grimsrud A, Stein DJ, Seedat $S$, et al. The association between hypertension and depression and anxiety disorders: results from a nationally-representative sample of South African adults. PLOS ONE 2009;4:e5552.

22. Msemburi W, Pillay-van Wyk V, Dorrington R, et al. Second National Burden of Disease Study for South Africa: cause-of-death profile for South Africa, 1997-2010. Cape Town: South African Medical Research Council, 2014. ISBN: 978-1-920618-34-6.

23. Bradshaw D, Msemburi W, Dorrington R, et al. HIV/AIDS in South Africa: how many people died from the disease between 1997 and 2010? AIDS 2016;30:771-8.

24. Mayosi BM, Benatar SR. Health and health care in South Africa-20 years after Mandela. N Engl J Med 2014;371:1344-53.

25. Mayosi BM, Lawn JE, van Niekerk A, et al. Health in South Africa: changes and challenges since 2009. Lancet 2012;380:2029-43.

26. Benatar SR, Mayosi BM. Health and health care in South Africa. N Engl J Med 2015;372:96.

27. American Psychiatric Association. Diagnostic and Statistical Manual of Mental Disorders (DSM-5). American Psychiatric Association, 2013.

28. World Health Organization. The global burden of disease, 2004 update. Geneva: World Health Organization, 2008.

29. American Psychiatric Association. Diagnostic and Statistical Manual of Mental Disorders, Text Revision (DSM-IV-TR). American Psychiatric Association, 2000.

30. American Psychiatric Association. Highlights of changes from $D S M-I V-T R$ to DSM-5. Arlington, VA: American Psychiatric Association, 2013. 
31. Paykel E, Andrade LH, Njenga F, et al. Changes needed in the classification of depressive disorders: options for ICD-11. World Psychiatry 2012;11(Suppl 1):37-42.

32. Sharp LK, Lipsky MS. Screening for depression across the lifespan: a review of measures for use in primary care settings. Am Fam Physician 2002;66:1001-8.

33. Ferrari AJ, Charlson FJ, Norman RE, et al. The epidemiological modelling of major depressive disorder: application for the Global Burden of Disease Study 2010. PLoS ONE 2013;8:e69637.

34. Vos T, Haby MM, Barendregt JJ, et al. The burden of major depression avoidable by longer-term treatment strategies. Arch Gen Psychiatry 2004;61:1097-103.

35. Porta M, Greenland S, Hernán M, et al. A dictionary of epidemiology. USA: Oxford University Press, 2014.

36. Lindert J, Ehrenstein OS, Priebe S, et al. Depression and anxiety in labor migrants and refugees-a systematic review and meta-analysis. Soc Sci Med 2009;69:246-57.

37. Fazel M, Wheeler J, Danesh J. Prevalence of serious mental disorder in 7000 refugees resettled in western countries: a systematic review. Lancet 2005;365:1309-14.

38. Norredam M, Garcia-Lopez A, Keiding N, et al. Risk of mental disorders in refugees and native Danes: a register-based retrospective cohort study. Soc Psychiatry Psychiatr Epidemiol 2009;44: 1023-9.

39. Steel Z, Chey T, Silove D, et al. Association of torture and other potentially traumatic events with mental health outcomes among populations exposed to mass conflict and displacement: a systematic review and meta-analysis. JAMA 2009;302:537-49.

40. Bandeira M, Higson-Smith $\mathrm{C}$, Bantjes $\mathrm{M}$, et al. The land of milk and honey: a picture of refugee torture survivors presenting for treatment in a South African trauma centre. Torture 2010;20:92-103.

41. Friedman ES, Anderson IM. Managing depression in clinical practice. London: Springer, 2010.
42. Hirschfeld RMA, Weissman MM. Risk factors for major depression and bipolar disorder. In: Davis KL, Charney D, Coyle JT, et al, eds. Neuropsychopharmacology - the fifth generation of progress. Philadelphia: Lippincott Williams \& Wilkins, 2002:1017-25.

43. Pillay-van Wyk V, Gbabe OF, Roomaney R, et al. Risk of bias tool for systematic review of observational studies. Society for Epidemiologic Research 2015 Annual Meeting 2015, 2015(1) http://epiresearch.org/poster/view_abstract.php?id=28237 (accessed 20 Sep 2015)

44. Hoy D, Brooks $\mathrm{P}$, Woolf $\mathrm{A}$, et al. Assessing risk of bias in prevalence studies: modification of an existing tool and evidence of interrater agreement. J Clin Epidemiol 2012;65:934-9.

45. Wells G, Shea B, O'Connell D, et al. The Newcastle-Ottawa Scale (NOS) for assessing the quality of nonrandomized studies in metaanalyses. 2009. http://www.ohri.ca/programs/clinical_epidemiology/ oxford.asp (accessed 29 Jun 2016).

46. Higgins JP, Thompson SG. Quantifying heterogeneity in a meta-analysis. Stat Med 2002;21:1539-58.

47. Higgins JPT, Thompson SG, Deeks JJ, et al. Measuring inconsistency in meta-analyses. BMJ 2003;327:557-60.

48. Egger M, Davey Smith G, Schneider M, et al. Bias in meta-analysis detected by a simple, graphical test. BMJ 1997;315:629-34.

49. Balshem $\mathrm{H}$, Helfand $\mathrm{M}$, Schunemann $\mathrm{HJ}$, et al. GRADE guidelines: 3. Rating the quality of evidence. J Clin Epidemiol 2011;64:401-6.

50. Guyatt GH, Oxman AD, Vist GE, et al. GRADE: an emerging consensus on rating quality of evidence and strength of recommendations. BMJ 2008;336:924-6.

51. Shamseer L, Moher D, Clarke M, et al. Preferred reporting items for systematic review and meta-analysis protocols (PRISMA-P) 2015: elaboration and explanation. BMJ 2015;349:g7647.

52. Moher D, Liberati A, Tetzlaff J, et al. Preferred reporting items for systematic reviews and meta-analyses: the PRISMA statement. Int J Surg 2010;8:336-41. 